\title{
Hidden Assumption of Gouy-Chapman-Stern Model
}

\author{
Chen Kun Li, ${ }^{1}$ Jun Huang ${ }^{1,2 *}$ \\ ${ }^{1}$ College of Chemistry and Chemical Engineering, Central South University, Changsha 410083, China. \\ ${ }^{2}$ School of Vehicle and Mobility, State Key Laboratory of Automotive Safety and Energy, Tsinghua University, Beijing 100084, China
}

\begin{abstract}
Understanding the double layer at the electrode-electrolyte interface is a long-standing challenge in electrochemistry. The orthodox Gouy-Chapman-Stern (GCS) model and its many derivatives invariably picture the double layer as a serial connection of a compact layer and a diffuse layer. We unravel herein that the serial connection tacitly prescribes a zero potential gradient at the solution-side boundary, which is, rigorously speaking, invalid. The bearing of this problematic assumption is pinpointed by comparing the double-layer impedance, which is analytically solved at the potential of zero charge, derived from the original and amended GCS models. Specifically, in the amended GCS model, the capacitance of the compact layer now shows frequency dispersion. The deviation between the original and amended models is greater when the double layer is confined in narrower space.
\end{abstract}

Research on the electrochemical double layer (EDL) has a long history dating back to the age of Helmholtz who viewed the EDL as a planar capacitor. Thereafter, a rich body of theoretical models have been developed. ${ }^{1-4}$ Amongst them, the Gouy-Chapman-Stern (GCS) model is quintessential. ${ }^{5-7}$ Improvements over the GCS model consider ion size, ${ }^{8-10}$ specific adsorption, ${ }^{11,12}$ solvent polarization, ${ }^{13-16}$ nonlocal short-range correlations ${ }^{17,18}$ and metal electronic effects. ${ }^{19-}$ 21

The GCS model is schematically illustrated in Fig. 1. The EDL is composed of a compact layer between the metal surface and the Helmholtz plane (HP), and a diffuse layer stretching toward the solution bulk. The HP is designated as the closest plane where solution species can approach the electrode surface, and the distance between the metal surface and the HP is usually of ca. several $\AA$. The diffuse layer is a non-electroneutral region where electrostatic interactions dominate over thermal motion, resulting in accumulation of counterions with charge of the sign opposite to the metal surface charge and depletion of coions with charge of the same sign. The diffuse layer has a characteristic thickness, termed the Debye length, $\lambda_{\mathrm{D}}=\sqrt{\epsilon_{\mathrm{s}} R T /\left(2 F^{2} c_{0}\right)}\left(\epsilon_{\mathrm{s}}\right.$ is the dielectric constant of the bulk solution, $c_{0}$ the bulk concentration, and other symbols have usual meanings), which is ca. $10 \mathrm{~nm}$ for an electrolyte solution with a concentration of $1 \mathrm{mM}$.

The impedance variant of the GCS model is shown in Fig. 1. Within the electric circuit representation, the EDL is modeled as a serial connection of a compact capacitor, $C_{\mathrm{H}}=\epsilon_{\mathrm{H}} / \delta_{\mathrm{H}}$ ( $\epsilon_{\mathrm{H}}$ and $\delta_{\mathrm{H}}$ are the permittivity and thickness of the space between the metal surface and the compact layer), and a diffuse layer part, $Z_{\mathrm{GC}}$. It follows naturally to write the double layer impedance as,

$$
Z_{\mathrm{dl}}=\left(j \omega C_{\mathrm{H}}\right)^{-1}+Z_{\mathrm{GC}}
$$

with $\omega$ being the angular frequency of the perturbation. Most often, $Z_{\mathrm{GC}}$ is simplified as the impedance of a pure capacitor, $Z_{\mathrm{GC}}=\left(j \omega C_{\mathrm{GC}}\right)^{-122}$, with the Gouy-Chapman capacitance given by,

$$
C_{\mathrm{GC}}=\frac{\epsilon_{\mathrm{s}}}{\lambda_{\mathrm{D}}} \cosh \left(\frac{U-U_{\mathrm{pzc}}}{2}\right)
$$

Here, $U=F \phi_{\mathrm{M}} / R T$ is the electrode potential normalized with respect to thermal voltage, and $U_{\text {pzc }}$ is the normalized potential of zero charge. Rigorously speaking, $Z_{\mathrm{GC}}$ may not be purely capacitive but show frequency dispersion ${ }^{23}$, which will become clear later.
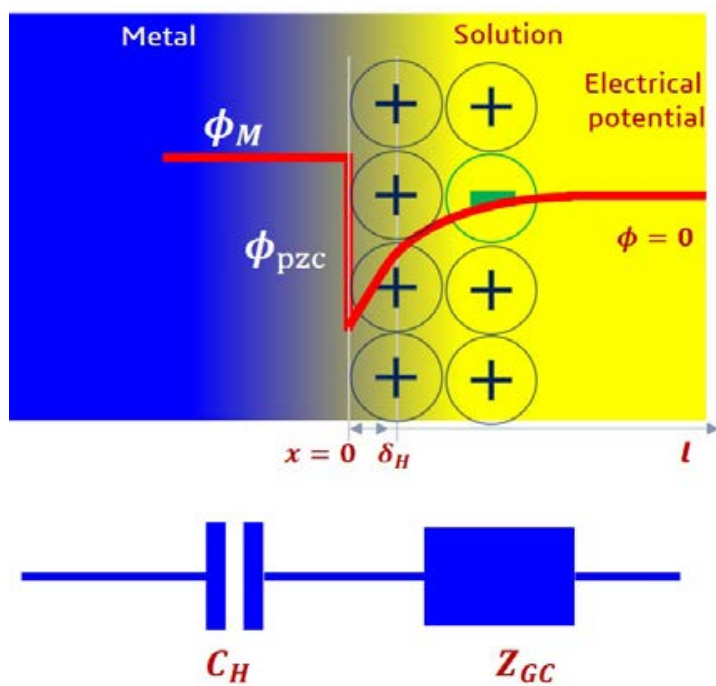

FIG. 1. Schematic illustration of the GCS double layer model and its equivalent electric circuit.

Herein, we will show that the orthodox relation given in Eq. (1) relies on a tacit assumption that the potential gradient at the solution-side boundary is zero, which is generally invalid. 
Removing this invalid assumption gives rise to a revised expression for the double-layer impedance which can be analytically obtained at the potential of zero charge. In the revised expression, the capacitance of the compact layer becomes frequency-dependent. Moreover, the difference in impedance response between the original model and the revised model becomes more significant when the double layer is confined in narrower space.

Consider the simplest case where the electrolyte is symmetrical, charge carriers are monovalent and have an identical diffusion coefficient, denoted $D$. The EDL is described by the Poisson-Nernst-Planck (PNP) theory in its standard form ${ }^{24}$,

$$
\begin{aligned}
& \frac{\partial C_{p}}{\partial \tau}=\frac{\partial}{\partial X}\left(\frac{\partial C_{p}}{\partial X}+C_{p} \frac{\partial U}{\partial X}\right) \\
& \frac{\partial C_{n}}{\partial \tau}=\frac{\partial}{\partial X}\left(\frac{\partial C_{n}}{\partial X}-C_{n} \frac{\partial U}{\partial X}\right) \\
& 0=\frac{\partial^{2} U}{\partial X^{2}}+\frac{1}{2}\left(C_{p}-C_{n}\right)
\end{aligned}
$$

Here, $C_{p}$ and $C_{n}$ are the concentration of positive and negative charge carriers referenced to the bulk concentration, $X$ is the spatial coordinate normalized with respect to $\lambda_{\mathrm{D}}, \tau=$ $t D / \lambda_{\mathrm{D}}^{2}$ is the dimensionless time.

The PNP theory is closed by following boundary conditions. In solution bulk, $X=L, C_{p}=C_{n}=1$ and the potential is taken as the reference, namely, $U=0$. At the HP, $X=0$, no reaction occurs, prescribing zero fluxes of positive and negative charge carriers there,

$$
\begin{aligned}
& \frac{\partial C_{p}}{\partial X}+C_{p} \frac{\partial U}{\partial X}=0 \\
& \frac{\partial C_{n}}{\partial X}-C_{n} \frac{\partial U}{\partial X}=0
\end{aligned}
$$

The potential at the HP is not an independent variable, but an implicit function of the electrode potential, $U_{\mathrm{M}}$, an independent variable in experiments, given by,

$$
\frac{\partial U}{\partial X}+\frac{\epsilon_{\mathrm{H}} \lambda_{\mathrm{D}}}{\epsilon_{\mathrm{s}} \delta_{\mathrm{H}}}\left(U_{\mathrm{M}}-U_{\mathrm{pzc}}-U\right)=0
$$

which is readily obtained from the notion that potential distribution in the compact layer is linear, and that potential gradients at the HP on two sides are correlated as, $\epsilon_{\mathrm{s}} \frac{\partial U}{\partial X}\left(X \rightarrow 0^{+}\right)=\epsilon_{\mathrm{H}} \frac{\partial U}{\partial X}\left(X \rightarrow 0^{-}\right)$; both are guaranteed by the Gauss law as there is no net charge in the compact layer or at the HP.

By Fourier transform, Eq. (3)-(5) can be analytically solved when the metal is initially held at $U_{\mathrm{pzc}}$, at which potential, $C_{p}=C_{n}=1$ and $U=0$. With algebra manipulations detailed in the Supporting Materials, we obtain the following analytical solution for the double-layer impedance,

$$
\begin{aligned}
& Z_{\mathrm{dl}} \\
& =\frac{1}{j \omega C_{\mathrm{H}}} \frac{1+\Omega}{1-\operatorname{sech}(L \sqrt{1+\Omega})} \\
& +\frac{1}{j \omega C_{\mathrm{GC}}^{0}} \frac{\frac{\tanh (L \sqrt{1+\Omega})}{\sqrt{1+\Omega}}+L \Omega}{1-\operatorname{sech}(L \sqrt{1+\Omega})}
\end{aligned}
$$

with $\Omega=j \omega \lambda_{\mathrm{D}}^{2} / D$ being the dimensionless imaginary frequency, and $C_{\mathrm{GC}}^{0}=\epsilon_{\mathrm{S}} / \lambda_{\mathrm{D}}$ the Gouy-Chapman capacitance at the pzc, see Eq.(2). To our best knowledge, this is the first time that the impedance response of a double layer described by the PNP theory is analytically solved. In the past, analytical impedance expressions were obtained under the electroneutrality assumption. ${ }^{25-28}$

In the low frequency limit, $\omega \rightarrow 0$, we simply Eq. (9) to,

$$
\begin{aligned}
Z_{\mathrm{dl}}= & \frac{1}{j \omega C_{\mathrm{H}}} \frac{1}{1-\operatorname{sech}(L)} \\
& +\frac{1}{j \omega C_{\mathrm{GC}}^{0}} \frac{\tanh (L)}{1-\operatorname{sech}(L)}
\end{aligned}
$$

which is further reduced back to the classical GCS model,

$$
Z_{\mathrm{dl}}=\frac{1}{j \omega C_{\mathrm{H}}}+\frac{1}{j \omega C_{\mathrm{GC}}^{0}}
$$

when $L \rightarrow \infty$, namely, when the solution phase is semiinfinite.

As the magnitudes of $Z_{d l}$ is infinite when $\omega \rightarrow 0$, we define a dimensionless complex capacitance as $C_{\mathrm{dl}}(\omega)=$ $\left(j \omega C_{\mathrm{H}}\left(Z_{\mathrm{dl}}(\omega)-R_{\mathrm{S}}\right)\right)^{-1}$, where $R_{\mathrm{s}}$ is the solution resistance, given by, $R_{\mathrm{s}}=Z_{\mathrm{dl}}(\omega \rightarrow \infty)=\lambda_{\mathrm{D}}^{2} / D C_{\mathrm{H}}+L \lambda_{\mathrm{D}}^{2} / D C_{\mathrm{GC}}^{0}$. The Nyquist plot of $C_{\mathrm{dl}}(\omega)$ is shown in Fig. 2, consisting of an arc in low frequency and an inclined line in high frequency. In the low frequency limit, $C_{\mathrm{dl}}(\omega)$ is asymptotic to $(1-\operatorname{sech}(\mathrm{L})) /(1+\xi \tanh (\mathrm{L}))$ obtained from Eq. (10), with $\xi=C_{\mathrm{H}} / C_{\mathrm{GC}}^{0}$, and in the high frequency limit, $C_{\mathrm{dl}}(\omega)$ is asymptotic to 1 .

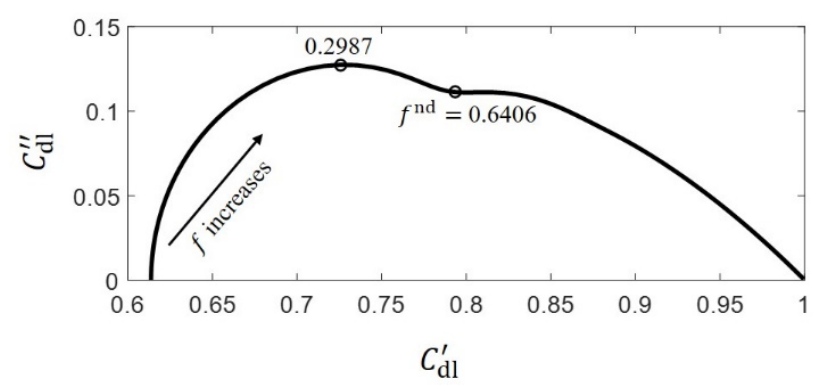

FIG. 2. The dimensionless complex capacitance obtained from Eq. (9), where $f^{\text {nd }}$ is the dimensionless frequency normalized with respect to $D / \lambda_{\mathrm{D}}^{2}$. Model parameters are: $c_{0}=0.1 \mathrm{~mol} / \mathrm{L}, \delta_{\mathrm{H}}=0.2 \mathrm{~nm}, L=5 \lambda_{\mathrm{D}}, D=1 \times$ $10^{-10} \mathrm{~m}^{2} / \mathrm{s}, \epsilon_{\mathrm{H}}=10 \epsilon_{0}, \epsilon_{\mathrm{s}}=80 \epsilon_{0}, \epsilon_{0}=8.85 \times$ 
$10^{-12} \mathrm{~F} / \mathrm{m}, F=96485 \mathrm{C} / \mathrm{mol}, N_{\mathrm{A}}=6.02 \times$

$10^{23} \mathrm{~mol}^{-1}, T=298 \mathrm{~K}, f=1 \times 10^{2} \mathrm{~Hz} \sim 1 \times 10^{13} \mathrm{~Hz}$

Compared to Eq. (1), Eq. (9) indicates that the contribution of the compact layer to $Z_{\mathrm{dl}}$ is not purely capacitive, expressed as $\left(j \omega C_{\mathrm{H}}\right)^{-1}$, but shows frequency dispersion, which can be described effectively by a frequency-dependent capacitance,

$$
C_{\mathrm{H}}^{\mathrm{eff}}=C_{\mathrm{H}} \frac{1-\operatorname{sech}(L \sqrt{1+\Omega})}{1+\Omega}
$$

which is asymptotic to $C_{\mathrm{H}}$ when $\omega \rightarrow 0$ and $L \rightarrow \infty$.

It now becomes clear that the oft-used equivalent electrical circuit based on the GCS model, depicted in Fig. 1, is, rigorously speaking, invalid. The underlying cause of this fundamental error lies in the boundary condition for the PNP equation that is tacitly assumed in the GCS model. The serial connection of the compact layer and the diffuse layer implies that,

$$
\frac{\epsilon_{\mathrm{H}}}{\delta_{\mathrm{H}}} \frac{\partial\left(\phi_{\mathrm{M}}-\phi_{\mathrm{pzc}}-\phi_{\mathrm{H}}\right)}{\partial t}=-\frac{\partial q_{\mathrm{GC}}}{\partial t}
$$

where $\phi_{\mathrm{H}}$ is the potential at the $\mathrm{HP}$ and $q_{\mathrm{GC}}$ the excess charge density stored in the diffuse layer.

Eq. (13) is transformed to,

$$
\frac{Q_{\mathrm{GC}}}{2}+\frac{\epsilon_{\mathrm{H}} \lambda_{\mathrm{D}}}{\epsilon_{\mathrm{s}} \delta_{\mathrm{H}}}\left(U_{\mathrm{M}}-U_{\mathrm{pzc}}-U\right)=0
$$

where $Q_{\mathrm{GC}}=2 q_{\mathrm{GC}} F \lambda_{\mathrm{D}} / R T \epsilon_{\mathrm{s}}$ is the normalized excess charge density in the diffuse layer. The boundary conditions expressed in Eq. (14) and (8) are equivalent only if,

$$
Q_{\mathrm{GC}}=2 \frac{\partial U}{\partial X}\left(X \rightarrow 0^{+}\right)
$$

which relies, as readily seen from Eq. (5), on the assumption that,

$$
\frac{\partial U}{\partial X}(X=L)=0
$$

However, a zero potential gradient at $X=L$, which is the middle plane of the solution phase, is not guaranteed, generally. From the PNP equation, the Fourier-transformed potential gradient at the $X=L$ is given by,

$$
\begin{aligned}
& \frac{\partial U}{\partial X}(X=L) \\
& =-U_{\mathrm{M}} \frac{\operatorname{sech}(L \sqrt{1+\Omega})+\Omega}{\frac{C_{\mathrm{GC}}^{0}}{C_{\mathrm{H}}} \sqrt{1+\Omega}+\frac{\tanh (L \sqrt{1+\Omega})}{\sqrt{1+\Omega}}+L \Omega}
\end{aligned}
$$

which becomes zero only when $\omega \rightarrow 0$ and $L \rightarrow \infty$. Otherwise, Eq. (16) is not satisfied and the boundary condition written in Eq. (14) is not correct.

The impedance expression with Eq. (14) as the boundary condition at the HP is written as,

$$
Z_{\mathrm{dl}}^{\mathrm{GCS}}=\frac{1}{j \omega C_{\mathrm{H}}}+\frac{1}{j \omega C_{\mathrm{GC}}^{0}} \frac{\frac{\tanh (L \sqrt{1+\Omega})}{\sqrt{1+\Omega}}+L \Omega}{1-\operatorname{sech}(L \sqrt{1+\Omega})}
$$

which varies from Eq. (9) by a difference of,

$$
\Delta Z=Z_{\mathrm{dl}}-Z_{\mathrm{dl}}^{\mathrm{GCS}}=\frac{1}{j \omega C_{\mathrm{H}}} \frac{\Omega+\operatorname{sech}(L \sqrt{1+\Omega})}{1-\operatorname{sech}(L \sqrt{1+\Omega})}
$$

which becomes zero only when $\omega \rightarrow 0$ and $L \rightarrow \infty$.

In the high frequency limit, $\omega \rightarrow \infty, \Delta Z$ is asymptotic to, $\Delta \mathrm{Z}(\omega \rightarrow \infty)=\lambda_{\mathrm{D}}^{2} / D C_{\mathrm{H}}$. Normalizing $\Delta Z(\omega \rightarrow \infty)$ with respect to the high-frequency asymptotic value of $Z_{\mathrm{dl}}$, $Z_{\mathrm{dl}}(\omega \rightarrow \infty)$ leads to,

$$
\Delta Z^{\mathrm{nd}}(\omega \rightarrow \infty)=(1+L \xi)^{-1}
$$

$\Delta Z^{\text {nd }}(\omega \rightarrow \infty)$ approaches zero when $L \rightarrow \infty$, and grows when $L$ decreases.

In the low frequency limit, $\omega \rightarrow 0, \Delta Z$ is asymptotic to, $\Delta Z(\omega \rightarrow 0)=\left(j \omega C_{\mathrm{H}}(\cosh (L)-1)\right)^{-1} \quad, \quad$ and the dimensionless counterpart normalized with respect to the high-frequency asymptotic value of $Z_{\mathrm{dl}}$ expressed in Eq. (10), reads,

$$
\Delta Z^{\mathrm{nd}}(\omega \rightarrow 0)=\frac{\operatorname{sech}(L)}{1+\xi \cdot \tanh (L)}
$$

which approaches zero when $L \rightarrow \infty$, and grows when $L$ decreases, namely, when the double layer is confined in narrower space.

Let us look at the difference between Eq. (9) and (18) in terms of the complex capacitance,

$$
\begin{aligned}
& \Delta C(\omega)=\frac{1}{j \omega C_{\mathrm{H}} Z_{\mathrm{dl}}^{\mathrm{GCS}}}-\frac{1}{j \omega C_{\mathrm{H}} Z_{\mathrm{dl}}} \\
& =\frac{1}{\left(j \omega C_{\mathrm{H}}\right)^{2} Z_{\mathrm{dl}}^{\mathrm{GCS}} Z_{\mathrm{dl}}} \frac{\Omega+\operatorname{sech}(L \sqrt{1+\Omega})}{1-\operatorname{sech}(L \sqrt{1+\Omega})}
\end{aligned}
$$

In the low frequency, namely, $\omega \rightarrow 0$,Eq. (22) is asymptotic to,

$$
\begin{aligned}
& \Delta C(\omega \rightarrow 0) \\
& =\frac{(1-\operatorname{sech}(L)) \operatorname{sech}(L)}{(1+\xi \cdot \tanh (L))(1-\operatorname{sech}(L)+\xi \cdot \tanh (L))}
\end{aligned}
$$

In the high frequency, namely, $\omega \rightarrow \infty$, Eq. (22) is asymptotic to zero.

The real part of Eq. (22), $\Delta C^{\prime}$, and the imaginary part, $-\Delta C^{\prime \prime}$, are shown in Fig. 3 as a function of the frequency. Two thicknesses of the double layer, $5 \lambda_{\mathrm{D}}$ and $50 \lambda_{\mathrm{D}}$, are compared. Two potentials, the pzc and $0.5 \mathrm{~V}$ more positive than the pzc, are compared. At potentials other than the pzc, analytical solution is currently unavailable, and numerical results are obtained. Implementation of the numerical calculation is provided in the Supplementary Materials. The accuracy of numerical solution is examined at the pzc. Analytical and numerical solutions agree well in low to mediate frequency, 
and noticeable deviation occurs at very high frequencies due to numerical errors.

At the pzc, as the frequency increases, $\Delta C^{\prime}$ first increases and then decreases, and $-\Delta C^{\prime \prime}$ shows a sinusoidal change. Comparing the curves of $5 \lambda_{\mathrm{D}}$ and $50 \lambda_{\mathrm{D}}$ at the pzc, FIG. 3 indicates that the magnitudes of $\Delta C^{\prime}$ and $\Delta C^{\prime \prime}$ are greater when the double layer is confined in narrower space. As the potential deviates away from the pzc, above features remain the same. It is noticed that $\Delta C^{\prime}$ at $0.5 \mathrm{~V}$ more positive than the pzc is higher than that at the pzc in low frequency, because there exists a steady-state charge distribution in the double layer, causing a larger difference of capacitance.

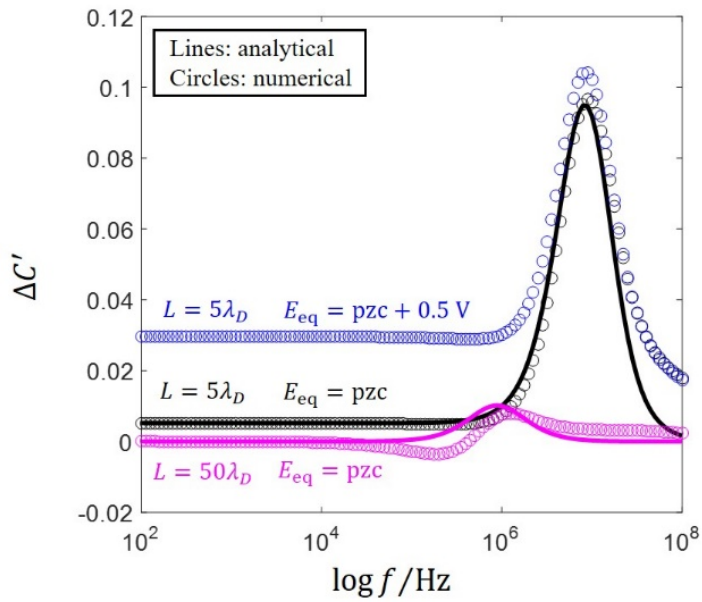

(a)

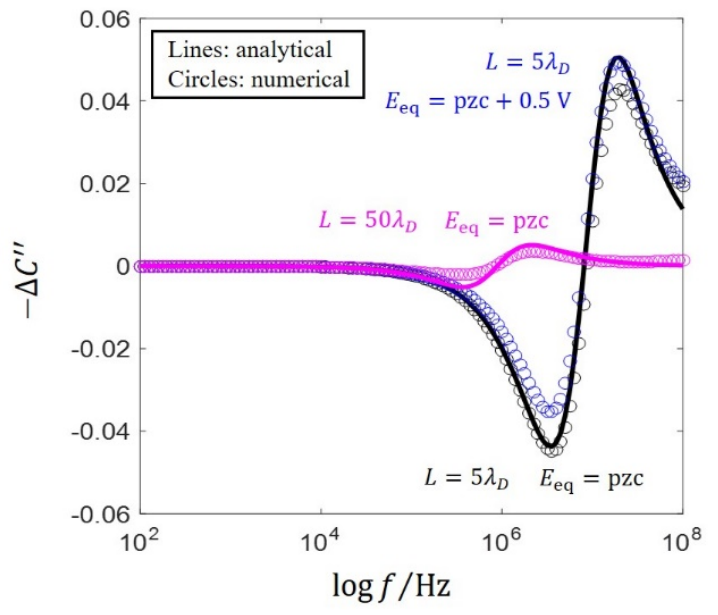

(b)

FIG. 3. Frequency dependency of the real (a) and imaginary (b) part of the dimensionless capacitance difference expressed in Eq. (22). The frequency range is $f=1 \times 10^{2} \mathrm{~Hz} \sim 1 \times 10^{8} \mathrm{~Hz}$. Model parameters are the same as those used in Fig. 2.
In conclusion, we have revealed that the serial connection involved in vast majority of double layer models requires that the potential gradient at the solution-side boundary is zero, which is generally invalid. This fundamental inconsistency has been amended, herein, resulting in a new expression of double-layer impedance with new features that change our understanding of the impedance response of the double layer.

This work was supported by National Natural Science Foundation of China (no. 21802170).

\section{Data availability statement}

The data that support the findings of this study are available from the corresponding author upon reasonable request.

*jhuangelectrochem@qq.com

\section{REFERENCES}

${ }^{1}$ B. B. Damaskin, and O. A. Petrii, J. Solid State Electrochem. 15, 1317 (2011)..

${ }^{2}$ D. C. Grahame, Chem. Rev. 41, 441 (1947).

${ }^{3}$ R. Parsons, Chem. Rev. 90, 813 (1990).

${ }^{4}$ M. Z. Bazant et al., Adv. Colloid and Interface Sci. 152, 48 (2009).

${ }^{5}$ G. Gouy, J. Phys. Theor. Appl. 9, 457 (1910).

${ }^{6}$ D. L. Chapman, Philos. Mag. 25, 475 (1913).

${ }^{7}$ O. Stern, Berichte der Bunsenges. Phys. Chem. 30, 508 (1924).

${ }^{8}$ A. A. Kornyshev, J. Phys. Chem. B 111, 5545 (2007).

${ }^{9}$ I. Borukhov, D. Andelman, and H. Orland, Phys. Rev. Lett. 79, 435 (1997).

${ }^{10}$ J. J. Bikerman, Trans. Faraday Soc. 35, 154 (1940).

${ }^{11}$ J. Huang et al., J. Phys. Chem. C 120, 13587 (2016).

${ }^{12}$ D.C. Grahame, and R. Parsons, J. Am. Chem. Soc. 83, 1291 (1961).

${ }^{13}$ A. Abrashkin, D. Andelman, and H. Orland, Phys. Rev. Lett. 99, 077801 (2007).

${ }^{14}$ A. Levy, D. Andelman, and H. Orland, Phys. Rev. Lett. 108, 227801 (2012).

${ }^{15}$ E. Gongadze, and A. Iglic, Bioelectrochem. 87, 199 (2012).

${ }^{16}$ J. O. M. Bockris, M. A. V. Devanathan, and K. Müller, Proc. R. Soc. London, Ser. A. Mathematical and Physical Sciences 274, 55 (1997).

${ }^{17}$ M. Bazant, B. D Storey, and A. Kornyshev, Phys. Rev. Lett. 106, 06102 (2011).

${ }^{18}$ R. Kjellander, J. Chem. Phys. 148, 193701 (2018).

${ }^{19}$ J. Huang, P. Li, and S. Chen, Phys. Rev. B 101, 125422 (2020).

${ }^{20}$ W.Schmickler, Chem. Rev. 96, 3177 (1996).

${ }^{21}$ A. A. Kornyshev, N. B. Luque, and W. Schmickler, J. Solid State Electrochem. 18, 1345 (2013).

${ }^{22}$ D.E. Smith, Anal. Chem. 48, 221A (1976).

${ }^{23}$ Z. Kerner, and T. Pajkossy, Electrochim. Acta 46, 207 (2000)

${ }^{24}$ M. Z. Bazant, K. Thornton, and A. Ajdari, Phys. Rev. E 70, 021506 (2004).

${ }^{25}$ J. Jamnik, and J. Maier, J. Electrochem. Soc. 146, 4183 (1999).

${ }^{26}$ J. Jamnik, and J. Maier, Phys. Chem. Chem. Phys. 3, 1668 (2001).

${ }^{27}$ W. Lai, and S. M. Haile, J. Am. Ceram. Soc. 88, 2979 (2005).

${ }^{28}$ J. R. Macdonald, J. Chem. Phys. 58, 4982 (1973). 